\title{
Dimensional overlap accounts for independence and integration of stimulus-response compatibility effects
}

\author{
Xun LiU \\ Mount Sinai School of Medicine, New York, New York \\ and Chinese Academy of Sciences, Beijing, China \\ AND \\ Yunsoo Park, Xiaosi Gu, AND Jin Fan \\ Mount Sinai School of Medicine, New York, New York
}

\begin{abstract}
Extensive studies have been conducted to examine various attentional control effects that stem from stimulusstimulus ( $\mathrm{S}-\mathrm{S}$ ) and stimulus-response ( $\mathrm{S}-\mathrm{R}$ ) incompatibility. Among these behavioral paradigms, the best-known are the Stroop effect, the Simon effect, and Posner's cue validity effect. In this study, we designed two behavioral tasks incorporating these effects (Simon-color-Stroop and Simon-spatial-Stroop) guided by a general framework of S-R ensemble, the dimensional overlap theory. We analyzed various attentional effects according to dimensional overlaps among S-S and S-R ensembles and their combinations. We found that behavioral performance was independently affected by various dimensional overlaps in the Simon-color-Stroop task, whereas different sources of dimensional overlap in the Simon-spatial-Stroop task interacted with each other. We argue that the dimensional overlap theory can be extended to serve as a viable unified theory that accounts for diverse attentional effects and their interactions and helps to elucidate neural networks subserving attentional control.
\end{abstract}

People encounter countless scenarios that require attentional control to resolve conflicts that originate from various stimulus-response ( $\mathrm{S}-\mathrm{R})$ incompatibilities. The best example in an experimental setting is the Stroop task (Stroop, 1935), which has become the "gold standard" in measuring attention since its inception (MacLeod, 1992). In a standard color-word Stroop task, individuals view a colored word and need to identify the word's ink color while ignoring its meaning. Because of the conflict between the task-relevant and task-irrelevant dimensions, incongruent trials (e.g., the word "red" in blue ink) require more attentional control than do neutral trials (e.g., the word "lot" in blue ink) in order to overcome the interferences. Many variants of the Stroop task have been developed (see a review in MacLeod, 1991) — for example, the spatial Stroop task, in which individuals are told to respond to the location of a word (Banich et al., 2000). Other variants involve numeric properties of the stimuli, such as counting or number comparison (Bush et al., 1998; Liu, Wang, Corbly, Zhang, \& Joseph, 2006).

The Stroop task usually requires attentional control to resolve conflict stemming from both stimulus-stimulus (S-S) and S-R incompatibilities. Another well-known paradigm, the Simon task (Simon \& Small, 1969), examines the incompatibility effect typically at the S-R level (see a review in Proctor \& Reeve, 1990). In the Simon task, an incompatible trial requires individuals to make a response that is spatially incompatible with the stimulus (e.g., respond with the right hand to a left-hand stimulus). The task-relevant stimulus attribute (e.g., color or shape) is not directly mapped onto a spatially defined response. This characteristic distinguishes the Simon task from the spatial Stroop task (see the detailed discussion below).

Attentional control is also important for its role in orienting, which is to direct attention to a spatial location, an object, or an attribute of a stimulus (Posner, Snyder, \& Davidson, 1980). After individuals are cued to orient to one spatial location (e.g., left of the fixation), performance is generally impaired when they have to disengage their attention from the cued location and shift toward a target that shows up at the opposite location (Posner, Cohen, $\&$ Rafal, 1982). Orienting of attention can be achieved through both task-directed instructions (endogenous) and salient external stimuli (exogenous). These two modes of attentional orienting are thought to relate to two partially segregated but interconnected attentional networks in the brain (Corbetta \& Shulman, 2002).

Extensive research on attention from diverse disciplines has produced many influential theories on the behavioral and neural mechanisms of attentional systems (Desimone \& Duncan, 1995; Knudsen, 2007; Posner \& Petersen, 1990). The aim of the present study was to build on a general S-R

X.Liu, liux@psych.ac.cn 
framework, dimensional overlap (DO), so as to provide a unified account for various attentional effects and further elucidate the behavioral and neural mechanisms of attention. DO was initially proposed as a model and taxonomy to account for various effects of S-R compatibility (Kornblum, Hasbroucq, \& Osman, 1990). It is defined as the similarity in perceptual, structural, and conceptual properties between stimulus sets, stimulus and response sets, or a combination of both. Given the task relevance of the stimulus properties, DO can refer to the similarity between the relevant $\left(\mathrm{S}_{\mathrm{R}}\right)$ and irrelevant $\left(\mathrm{S}_{\mathrm{I}}\right)$ stimuli (or relevant and irrelevant dimensions of the same stimulus; $S_{R}-S_{I}$ overlap), the $S_{R}$ and response $\left(S_{R}-R\right.$ overlap), and the $S_{I}$ and response $\left(S_{I}-R\right.$ overlap) (Kornblum et al., 1990; Kornblum \& Lee, 1995; Kornblum, Stevens, Whipple, \& Requin, 1999; Zhang \& Kornblum, 1998). For any S-R ensembles that consist of all three components (relevant stimulus, irrelevant stimulus, and response), DO can occur independently between any of the two components, thus giving rise to eight different classes of potential S-R ensembles.

On the basis of this taxonomy, DO captures various attentional effects under a unified framework. For example, a typical Simon task usually involves responding to a stimulus presented on either the left or the right with a left or right buttonpress. The required response is often associated with another nonspatial property of the stimulus specified by the task (e.g., press the left key for a blue color), and thus, the location of the stimulus is task irrelevant. Therefore, there is only dimensional overlap between $S_{I}$ and $R$, whereas no overlap exists between $S_{R}$ and $S_{I}$ or between $S_{R}$ and $R$. This is classified as a Type 3 S-R ensemble (Kornblum et al., 1990). On the other hand, a typical Stroop task often involves dimensional overlap across all three dimensions, which exemplifies a Type 8 $\mathrm{S}-\mathrm{R}$ ensemble. For example, when people are asked to name the ink color of the words and the words are color related (e.g., the word "blue" in red ink), $\mathrm{S}_{\mathrm{R}}$ (ink color) and $\mathrm{S}_{\mathrm{I}}$ (color word) dimensions overlap with each other, and they both overlap with R (color naming). DO can also be extended to account for the orienting effect of Posner's cue validity task, by introducing dimensional overlap between locations of the cue and target. We consider this as another type of S-S compatibility, because participants are usually required to respond to a property of the target while disregarding the location of the cue or the target.

The present study examined diverse attentional effects within the same group of participants, with two experiments incorporating the Simon, the Stroop, and Posner's cue validity effects. Experiment 1 used a Simon-colorStroop task (see Figure 1), in which participants responded to the color of a painted triangle within a white diamond shape while ignoring the word printed in the shape and the pointing direction of the colored triangle. Experiment 2 used a Simon-spatial-Stroop task (see Figure 2), in which participants responded to an arrow pointing either upward or downward, using a left or right button following a valid or an invalid spatial cue. The present study design bears the following technical and theoretical significance. First, with theory-driven task designs, we can directly compare and correlate various attentional effects so as to test hy- potheses with regard to independence or interaction of these effects as a result of S-S and S-R dimensional overlaps. Second, on the basis of the behavioral findings for these tasks, we can generate and further test hypotheses with regard to the common and distinct attentional control networks in the brain. Third, by administering these two tasks in the same sample of participants, we can rule out confounding due to differences between samples and between design manipulations across studies.

On the basis of the DO framework, we hypothesized that given different sources of dimensional overlap, S-S and S-R compatibility are separately detected and processed by two distinct conflict-monitoring modules. In addition, compatibility effects originating from various $\mathrm{S}-\mathrm{S}$ and $\mathrm{S}-\mathrm{R}$ dimensional overlaps are integrated and processed by a common module of executive control (Model 2CM1EC). Therefore, we predicted that behavioral performance would be independently affected by an $\mathrm{S}-\mathrm{S}$ or S-R compatibility effect when only one source is present. When both S-S and S-R compatibility effects exist, they will interact with each other as follows: Their effects will be additive when both of them yield facilitation or interference; their effects will cancel out when they have opposite effects. Two alternative models exist: One assumes distinct modules for both conflict monitoring and executive control for S-S and S-R compatibility effects (Model 2CM2EC); another assumes a common module for conflict detection for both S-S and S-R compatibility effects and a common module for executive control (Model 1CM1EC). Model 2CM2EC predicts a subadditive effect of S-S and S-R compatibility, because they are processed separately with no potential interaction, and thus, behavioral performance will be determined by a horse race of these two separate effects; Model 1CM1EC predicts a superadditive effect of S-S and S-R compatibility, given that both are competing for a common processing resource.

\section{METHOD}

\section{Participants}

Thirty healthy adults (18-40 years old, average of $25 \pm 2$; 15 women) participated in the study. Informed written consent forms approved by the Mount Sinai School of Medicine institutional review board office were obtained from the participants.

\section{Tasks and Stimulus Materials}

The participants completed two different experiments. One experiment used a Simon-color-Stroop task (see Figure 1), and the other experiment used a Simon-spatial-Stroop task (see Figure 2). The order of the tasks was counterbalanced across participants.

Simon-color-Stroop task. A modified version of the Simoncolor-Stroop task (Kornblum et al., 1999) was used. The participants were trained to respond to two colors (red and blue) by pressing left/right buttons. For example, during training, they were asked to press the left button for the blue color and the right button for the red color of a fully colored diamond shape. During the test, half of the diamond shape (a triangle) was painted in either red or blue (Figure 1A). The pointing angle of the painted triangle (the right angle) can point to one of the four directions (left, right, up, and down). A neutral (e.g., "door"), congruent (e.g., "blue"), or incongruent (e.g., "red") word, with regard to the color of the triangle (e.g., blue), was overlaid in the center of the diamond. The participants were asked to respond to the color of the triangle according to the rules they were 
A

\section{Congruent}

Incongruent
Type 1*
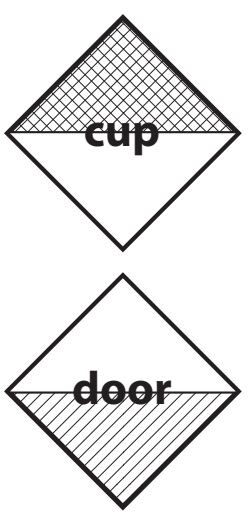

$Q$

Response
Dimensional
Overlap

B

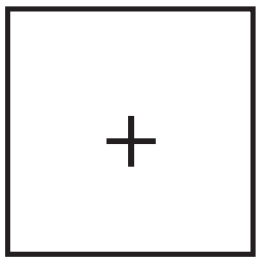

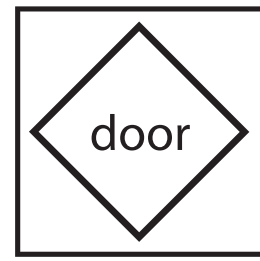
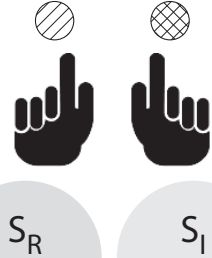

color

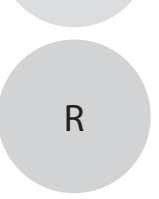

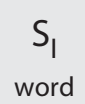

$S_{1}$

direction

\section{$100 \mathrm{msec}$}

\begin{abstract}
$200 \mathrm{msec}$
\end{abstract}
Type 3
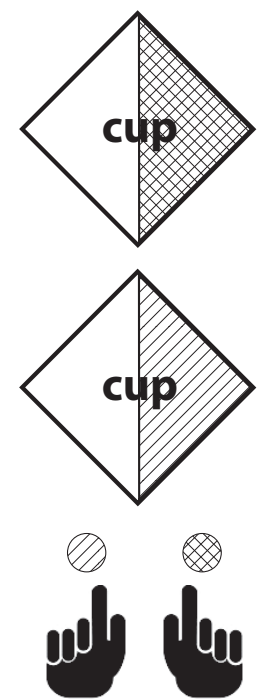

$\mathrm{S}_{\mathrm{R}}$

color
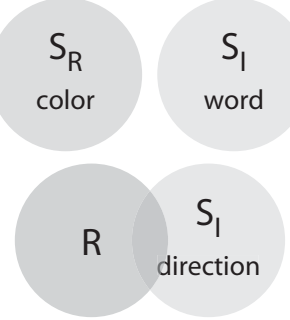

Type 4
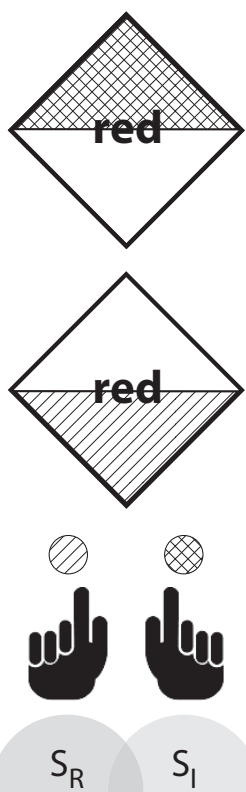

$S_{R}$

color

\section{$\mathrm{S}_{1}$}

word

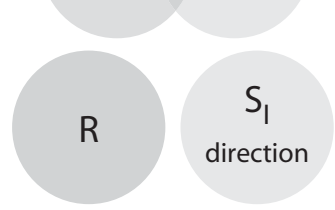

Type 7
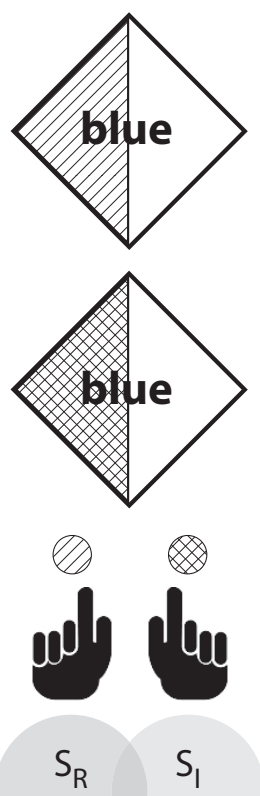

color word

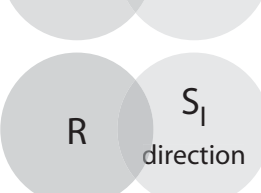

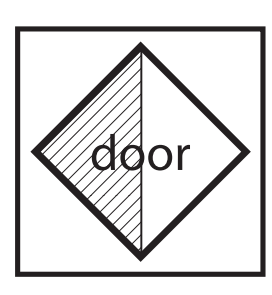

$1,200 \mathrm{msec}$

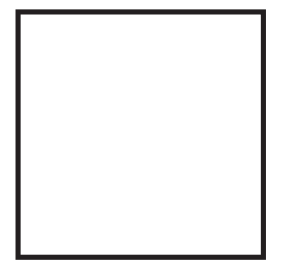

$500 \mathrm{msec}$

Figure 1. Experimental designs (A) and trial procedures (B) for the Simon-color-Stroop task. (A) Stimuli of the Simon-color-Stroop task categorized as different types of dimensional overlap. The lattice shade denotes the red color, and the diagonal shade denotes the blue color. $S_{R}$, task-relevant stimulus dimension; $S_{I}$, task-irrelevant stimulus dimension; $R$, response dimension. The Type 1 condition consisted of neutral trials. Therefore, the labels "Congruent" and "Incongruent" do not apply to the trials. (B) After the fixation, a word was shown for 200 msec prior to the onset of the colored triangle to enhance the interference effect of the word.

trained with, while ignoring both the word and the pointing angle of the colored triangle. Therefore, the task-relevant dimension was the color, and the task-irrelevant dimensions were the word and the pointing angle of the colored triangle.

The trials were classified into different conditions, based on the presence and nature of the conflict (e.g., S-S or S-R) caused by the task-irrelevant dimension (Figure 1A). For example, when the overlaying word is neutral (e.g., "door") and the pointing direction of the colored triangle is either up or down, the stimulus is considered a neutral trial (NEU; Type $1 \mathrm{~S}-\mathrm{R}$ ensemble), since both task-irrelevant dimensions (i.e., the word and pointing direction) are neutral with regard to the task-relevant dimension (i.e., the color) and response (i.e., left or right). When one of the task-irrelevant dimensions or both overlap with the task-relevant dimension and response, the trial can be considered either congruent or incongruent in terms of S-R ensembles. The second and third columns in Figure 1A illustrate the trials on which one of the task-irrelevant dimensions overlaps with the task-relevant dimension, whereas the last column in Figure $1 \mathrm{~A}$ illustrates the trials on which both task-irrelevant dimensions overlap with the task-relevant dimension. For example, when the word is neutral (e.g., "door") and the colored triangle is pointing to either the left or the right, this creates the $S-R$ ensemble of compatibility (SRC and SRI; Type 3), with the left- and right-pointing triangles overlapping with the left and right responses to the colors. When the colored triangle is pointing either up or down, the pointing direction is neutral with regard to the response (i.e., left and right); therefore, only the color word (i.e., "red" or "blue") overlaps with the color of the triangle, which results in the $S-S$ ensemble of compatibility (SSC and SSI; Type 4). When both task-irrelevant dimensions overlap with the task-relevant feature and response, com- 
patibility of the $\mathrm{S}-\mathrm{S}$ or $\mathrm{S}-\mathrm{R}$ ensemble is independently manipulated to create trials (SSCSRC, SSCSRI, SSISRC, and SSISRI; Type 7). Each participant completed three runs of this task. Each run consisted of 120 trials, with a trial lasting $2 \mathrm{sec}$ (Figure 1B). Nine types of stimuli (NEU, SSC, SSI, SRC, SRI, SSCSRC, SSCSRI, SSISRC, and SSISRI) were organized in two types of blocks. One block of 20 trials consisted of equal number of NEU, SSC, SSI, SRC, and SRI, and the other block of 20 trials consisted of equal number of NEU, SSCSRC, SSCSRI, SSISRC, and SSISRI, with trials in both blocks randomly mixed.

Simon-spatial-Stroop task. A modified Simon-spatial-Stroop task (Liu, Banich, Jacobson, \& Tanabe, 2004) was administered. The participants were trained to respond to an upward or downward arrow by pressing one of the two buttons arranged from left to right. For example, they were asked to press the left button for the up- ward arrow and the right button for the downward arrow. During the test, the arrow was presented at one of the five possible locationscenter, left, right, top, and bottom - as shown in Figure 2.

The task-relevant dimension was the direction of the arrow, whereas the cue location and target location were the task-irrelevant dimensions, which should be ignored or inhibited in the case of a conflict between the task-relevant and task-irrelevant dimensions. Similar to the Simon-color-Stroop task above, the trials were classified into different conditions, based on the presence and nature of the conflict (e.g., S-S or S-R) caused by task-irrelevant dimensions (Figure 2A). For example, when both the cue and target were presented in the center, the trial was considered to be neutral, since the locations of the cue and target did not overlap or conflict with the response to the target (NEU; Type 1). The second column in the figure represents the dimensional overlap between the task-irrelevant dimension (cue and
A

Congruent

Incongruent

Response

imensional Overlap
Type 1*
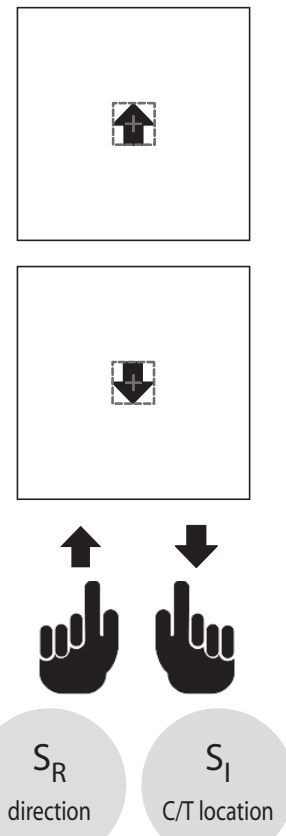

$\mathrm{R}$
Type 3
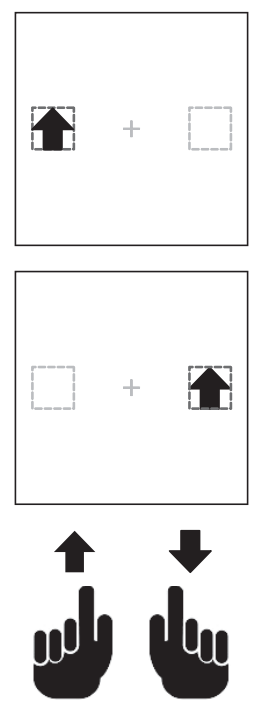

$S_{R}$

direction

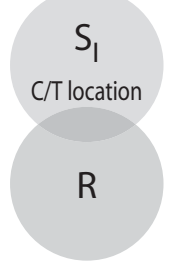

Type 4
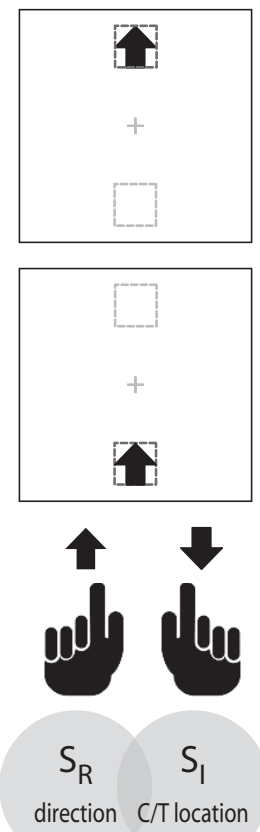

$\mathrm{R}$
Type 7
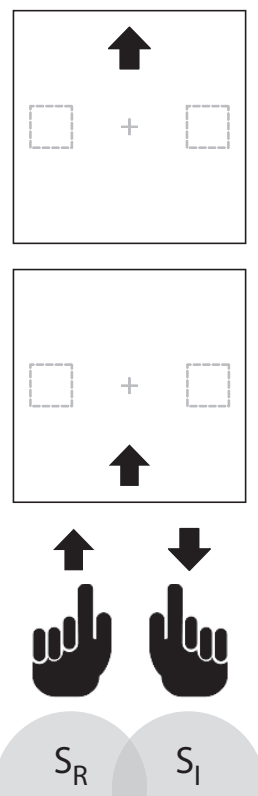

direction Tlocation

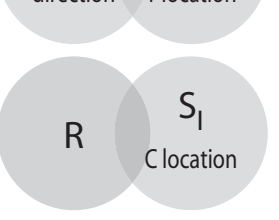

B

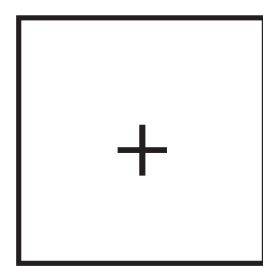

$100 \mathrm{msec}$

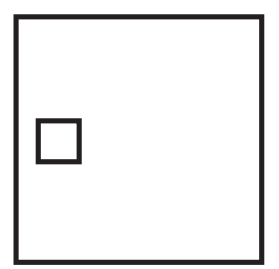

$200 \mathrm{msec}$

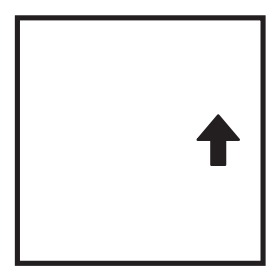

$1,200 \mathrm{msec}$

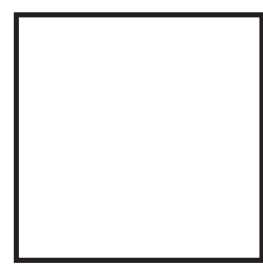

$500 \mathrm{msec}$

Figure 2. Experimental designs (A) and trial procedures (B) for the Simon-spatial-Stroop task. (A) Stimuli of the Simon-spatialStroop task categorized as different types of dimensional overlap. A dashed square indicates the location of the cue presented for $200 \mathrm{msec}$ before the target onset, with the color indicating validity of the cue: valid, dark gray; invalid, light gray. This is just for illustration purpose. In the actual experiment, the cue was always a solid white frame against a dark background. (B) After the fixation, a square was presented as a spatial cue for $\mathbf{2 0 0} \mathbf{m s e c}$ prior to the onset of the target arrow. 
target locations) and response in terms of the S-R ensemble (SRC and SRI; Type 3), since the left and right locations of the cue or target overlapped with the left and right responses but did not overlap with the task-relevant dimension (i.e., upward or downward arrow) of the stimulus. The next column represents the dimensional overlap between the task-relevant dimension and task-irrelevant dimension in terms of the S-S ensemble (SSC and SSI; Type 4), since the locations (i.e., top or bottom) of the cue or target overlapped with the directions (i.e., upward or downward) of the arrow, but not with the left or right responses. Figure 2A also shows a Type 7 ensemble, in which there exist dimensional overlaps between the task-relevant dimension (target direction) and one task-irrelevant dimension (target location), as well as between the other task-irrelevant dimension (cue location) and response. We did not include the Type 7 ensemble in the present study.

When the cue was in the center, the target was always presented in the center. This created a neutral baseline condition. On the remaining trials, the target was presented at the same location as the cue $67 \%$ of the time (valid cue, C-T compatible or CTC) and at the opposite location from the cue $33 \%$ of the time (invalid cue, $\mathrm{C}-\mathrm{T}$ incompatible or CTI). On half of these trials (excluding neutral stimuli), the target location was congruent with the direction of the target (SSC; e.g., an upward arrow on the top) or the response toward the target (SRC; e.g., left buttonpress for an upward arrow on the left). The other half consisted of the incongruent trials on which either the location of the target was incongruent (SSI; e.g., an upward arrow on the bottom) or the response toward the target was incongruent (SRI; e.g., left buttonpress for an upward arrow on the right). Each participant completed three runs of this task. Each run consisted of 120 trials, with a trial lasting $2 \mathrm{sec}$ (Figure 2B). Nine types of stimuli (NEU, CTCSSC, CTCSSI, CTISSC, CTISSI, CTCSRC, CTCSRI, CTISRC, and CTISRI) were organized in two types of blocks. One block of 20 trials consisted of NEU ( 2 trials), CTCSSC (6 trials), CTCSSI ( 6 trials), CTISSC ( 3 trials), and CTISSI ( 3 trials), and the other block of 20 trials consisted of NEU ( 2 trials), CTCSRC ( 6 trials), CTCSRI (6 trials), CTISRC (3 trials), and CTISRI (3 trials), with trials in both blocks randomly mixed.

\section{RESULTS}

The response times (RTs) and accuracy for the Simoncolor-Stroop and Simon-spatial-Stroop tasks are listed in the Appendix. The effects of interest are summarized below.

\section{Simon-Color-Stroop Task}

Nine conditions can be viewed as a factorial combination of S-S and S-R compatibility. Two 3 (S-S: congruent, neutral, and incongruent $) \times 3(\mathrm{~S}-\mathrm{R}$ : congruent, neutral, and incongruent) repeated measures ANOVAs were conducted to examine the main effects of S-S and S-R compatibility and their interaction on RT and accuracy, respectively. For RT, there were main effects for both $\mathrm{S}-\mathrm{S}$ and $\mathrm{S}-\mathrm{R}$ compatibility effects $\left[\mathrm{S}-\mathrm{S}, F(2,58)=29.95, M S_{\mathrm{e}}=523, p<.001 ; \mathrm{S}-\mathrm{R}\right.$, $\left.F(2,58)=69.04, M S_{\mathrm{e}}=538, p<.001\right]$. The interaction was not significant $\left[F(4,116)=1.36, M S_{\mathrm{e}}=332, p>.05\right]$, indicating an additive effect between S-S and S-R compatibility (see Figure 3A). For accuracy, there were main effects for both S-S and S-R compatibility effects [S-S, $F(2,58)=5.32, M S_{\mathrm{e}}=6, p<.01 ; \mathrm{S}-\mathrm{R}, F(2,58)=11.87$, $\left.M S_{\mathrm{e}}=14, p<.001\right]$. The interaction was not significant $\left[F(4,116)=1.01, M S_{\mathrm{e}}=5, p>.05\right.$; see Figure 3B $]$. Accuracy across conditions was negatively associated with RT, ruling out a speed-accuracy trade-off effect on RT.

The next step was to separately examine the facilitation and interference caused by S-S and S-R compatibility ef- fects. We obtained the facilitation and interference effects by subtracting the RT in the neutral condition (NEU) from that in the congruent (e.g., SSC) and incongruent (e.g., SSI) conditions. A negative value indicates the facilitation effect (shorter RT), and a positive value shows the interference effect (longer RT). As is shown in Figure 4A, when there existed only one source of dimensional overlap (either S-S or S-R), both facilitation (for the congruent conditions) and interference (for the incongruent conditions) effects were significant (Bonferroni correction at $p<.05)$. The facilitation by the congruent S-S overlap yielded an improvement of $20 \mathrm{msec}[t(29)=4.86, p<$ $.001]$; RT was shortened $17 \mathrm{msec}$ by the congruent $\mathrm{S}-\mathrm{R}$ overlap $[t(29)=4.18, p<.001]$. The interference by the incongruent S-S overlap lengthened RT by $14 \mathrm{msec}$ $[t(29)=2.78, p<.01]$; the incongruent S-R overlap lengthened RT by $22 \mathrm{msec}[t(29)=4.11, p<.001]$.

When both $\mathrm{S}-\mathrm{S}$ and $\mathrm{S}-\mathrm{R}$ overlaps were manipulated, both facilitation and interference effects of S-S and S-R overlaps showed additive effects. Double facilitation (SSCSRC vs. NEU) yielded a reduction in RT by $34 \mathrm{msec}$ $[t(29)=8.31, p<.001]$; it was not significantly different from the sum $(36 \mathrm{msec})$ of single facilitation effects of S-S (SSC vs. NEU) and S-R (SRC vs. NEU) overlaps $[t(29)=0.48, p>.05]$. Double interference (SSISRI vs. $\mathrm{NEU})$ caused an increase in RT of $31 \mathrm{msec}[t(29)=6.99$, $p<.001]$; it was not significantly different from the sum (35 msec) of single interference effects of S-S (SSI vs. NEU) and S-R (SRI vs. NEU) overlaps $[t(29)=0.61$, $p>.05]$.

Obviously, when interference and facilitation were stacked together by comparing the incongruent and congruent conditions (e.g., SSI vs. SSC), the effects were significant [for the S-S congruency effect (SSI vs. SSC), $t(29)=$ 5.99, $p<.001$; for the S-R congruency effect (SRI vs. $\mathrm{SRC}), t(29)=6.14, p<.001]$. When there was a crossover between S-S and S-R overlap congruency (e.g., SSCSRI or SSISRC), the facilitation and interference effects arising from $\mathrm{S}-\mathrm{S}$ and $\mathrm{S}-\mathrm{R}$ overlaps were competing against each other and canceling out each other's effect. It appeared that the S-R interference effect overpowered the S-S facilitation effect [SSCSRI vs. NEU, $t(29)=3.31, p<.005$ ], whereas the S-R facilitation effect canceled out the S-S interference effect [SSISRC vs. NEU, $t(29)=1.21, p>.05]$. None of the effects - S-S, S-R, as well as dual S-S and S-R-was significantly correlated with the others, which implies relative independence of each effect.

\section{Simon-Spatial-Stroop Task}

Excluding the NEU condition, the remaining eight conditions can be viewed as two factorial combinations of cue-target validity $(\mathrm{C}-\mathrm{T})$ and a Simon $(\mathrm{S}-\mathrm{R})$ or spatial Stroop (S-S) effect. Two 2 (C-T: congruent and incongruent $) \times 2(\mathrm{~S}-\mathrm{R}$ : congruent and incongruent) repeated measures ANOVAs were conducted for the Simon effect on RT and accuracy, respectively. For $\mathrm{C}-\mathrm{T} \times \mathrm{S}-\mathrm{R}$ analysis on the RT, there were main effects for both $\mathrm{C}-\mathrm{T}$ and $\mathrm{S}-\mathrm{R}$ compatibility effects $\left[\mathrm{C}-\mathrm{T}, F(1,29)=24.05, M S_{\mathrm{e}}=\right.$ $775, p<.001$; S-R, $F(1,29)=38.42, M S_{\mathrm{e}}=908, p<$ $.001]$. The interaction was not significant $[F(1,29)=1.11$, 
A Behavioral Performance of the Simon-Color-Stroop Task

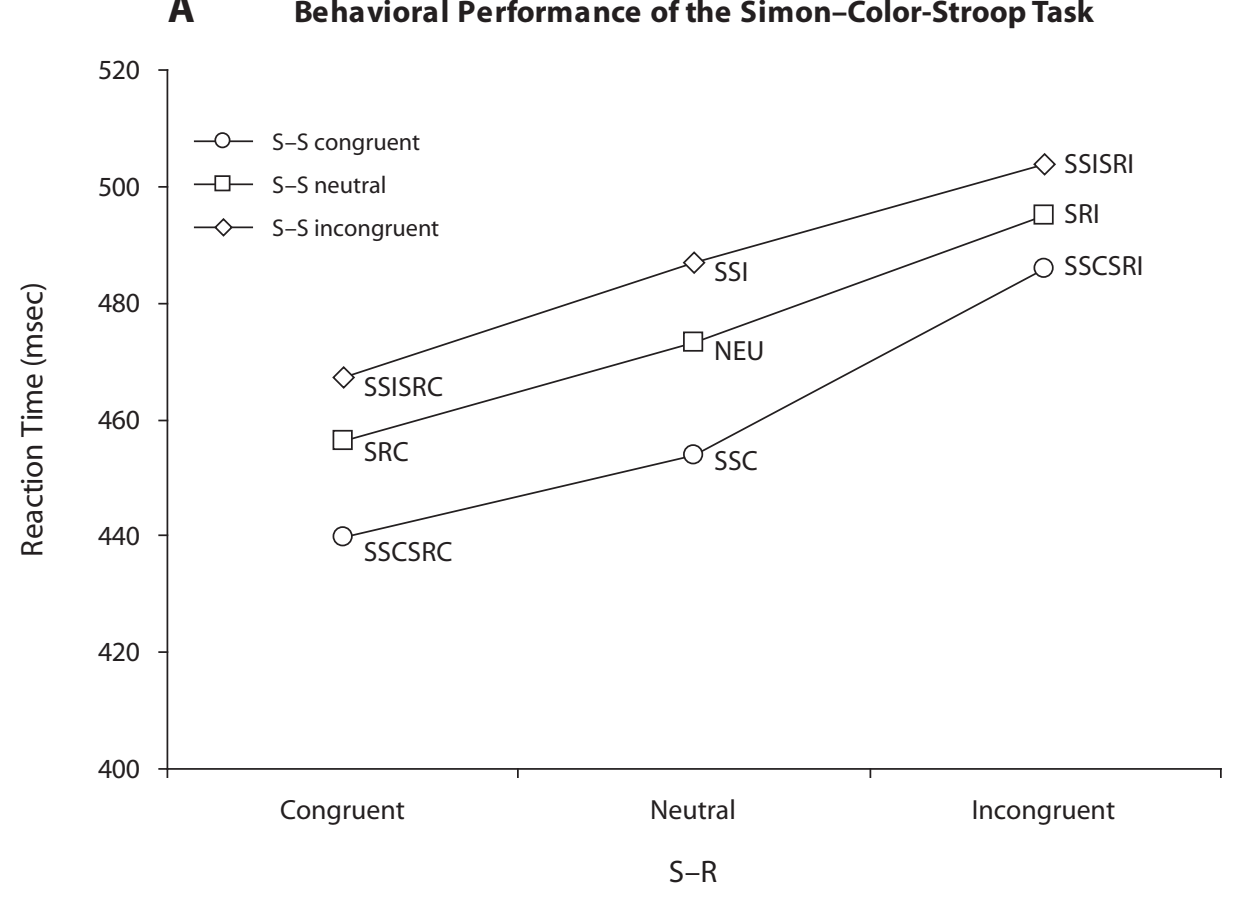

B

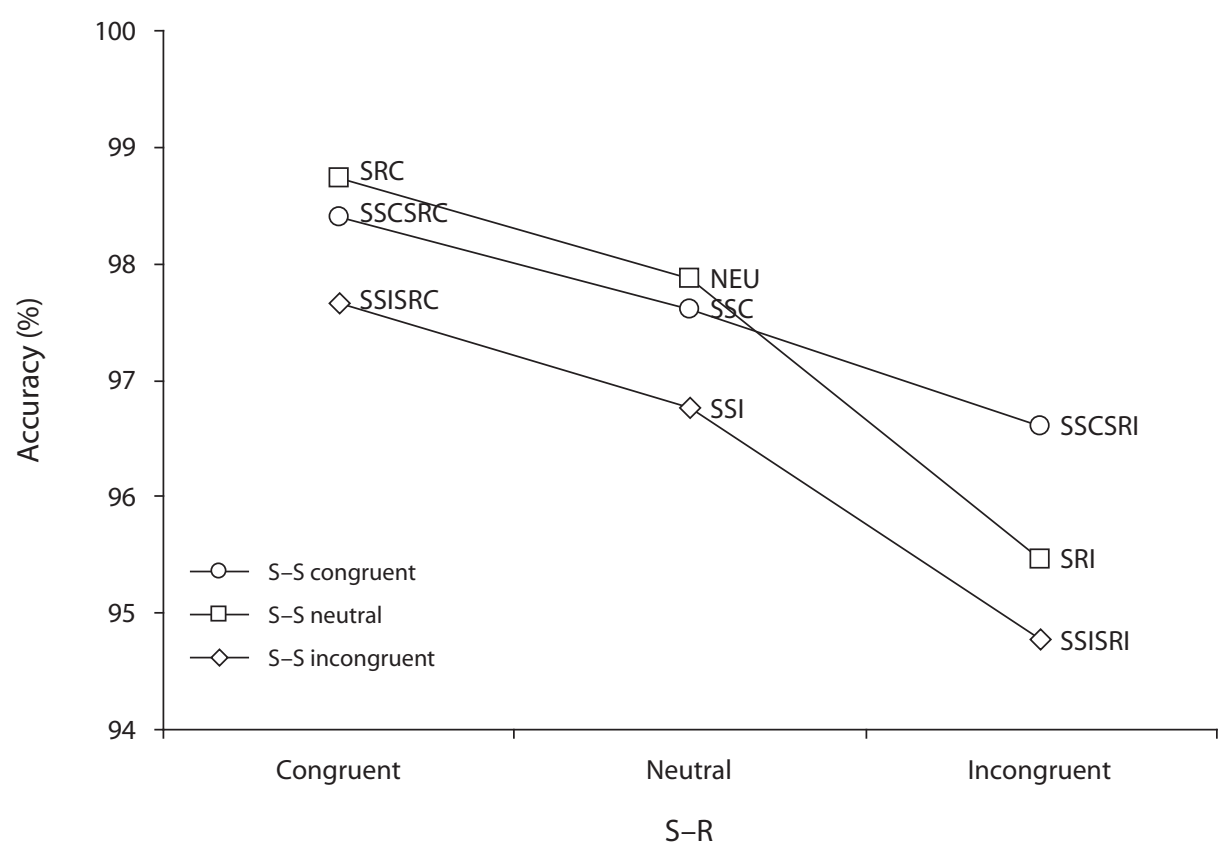

Figure 3. Reaction times (A) and accuracy (B) on the Simon-color-Stroop task.

$\left.M S_{\mathrm{e}}=664, p>.05\right]$, indicating an additive effect between the $\mathrm{C}-\mathrm{T}$ and $\mathrm{S}-\mathrm{R}$ compatibility (Figure $5 \mathrm{~A}$ ). For $\mathrm{C}-\mathrm{T} \times$ $\mathrm{S}-\mathrm{R}$ analysis on accuracy, there were main effects for both C-T and S-R compatibility effects [C-T, $F(1,29)=4.40$, $M S_{\mathrm{e}}=16, p<.05 ; \mathrm{S}-\mathrm{R}, F(1,29)=31.16, M S_{\mathrm{e}}=18, p<$ $.001]$. The interaction was not significant $[F(1,29)=0$, $p>.05]$, indicating an additive effect between the $\mathrm{C}-\mathrm{T}$ and S-R compatibility (Figure 5B).
Two additional 2 (C-T: congruent and incongruent) $\times$ 2 (S-S: congruent and incongruent) repeated measures ANOVAs were conducted for the spatial Stroop effect on RT and accuracy, respectively. For $\mathrm{C}-\mathrm{T} \times \mathrm{S}-\mathrm{S}$ analysis on $\mathrm{RT}$, there were main effects for both $\mathrm{C}-\mathrm{T}$ and $\mathrm{S}-\mathrm{S}$ compatibility effects [C-T, $F(1,29)=39.87, M S_{\mathrm{e}}=819, p<$ .001 ; S-S, $\left.F(1,29)=18.11, M S_{\mathrm{e}}=731, p<.001\right]$. The interaction was also significant $\left[F(1,29)=6.22, M S_{\mathrm{e}}=\right.$ 
A Behavioral Performance of the Simon-Spatial-Stroop Task
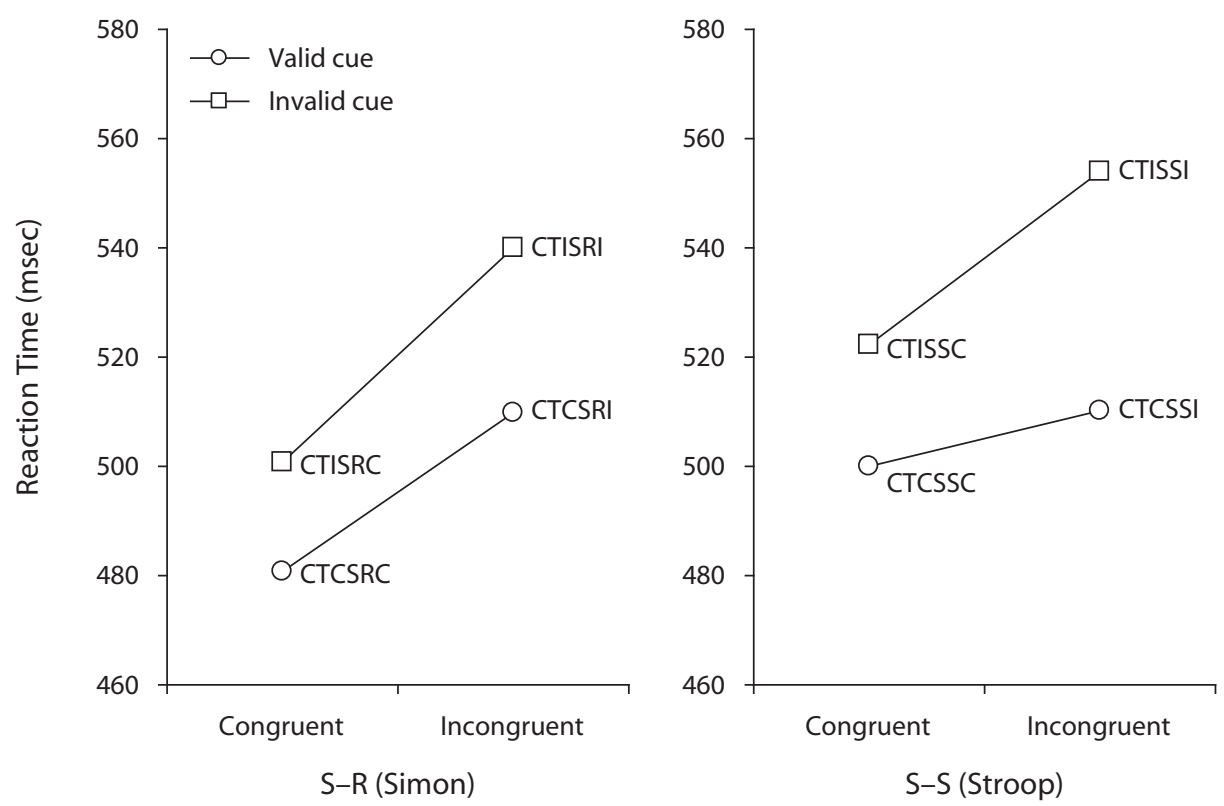

B
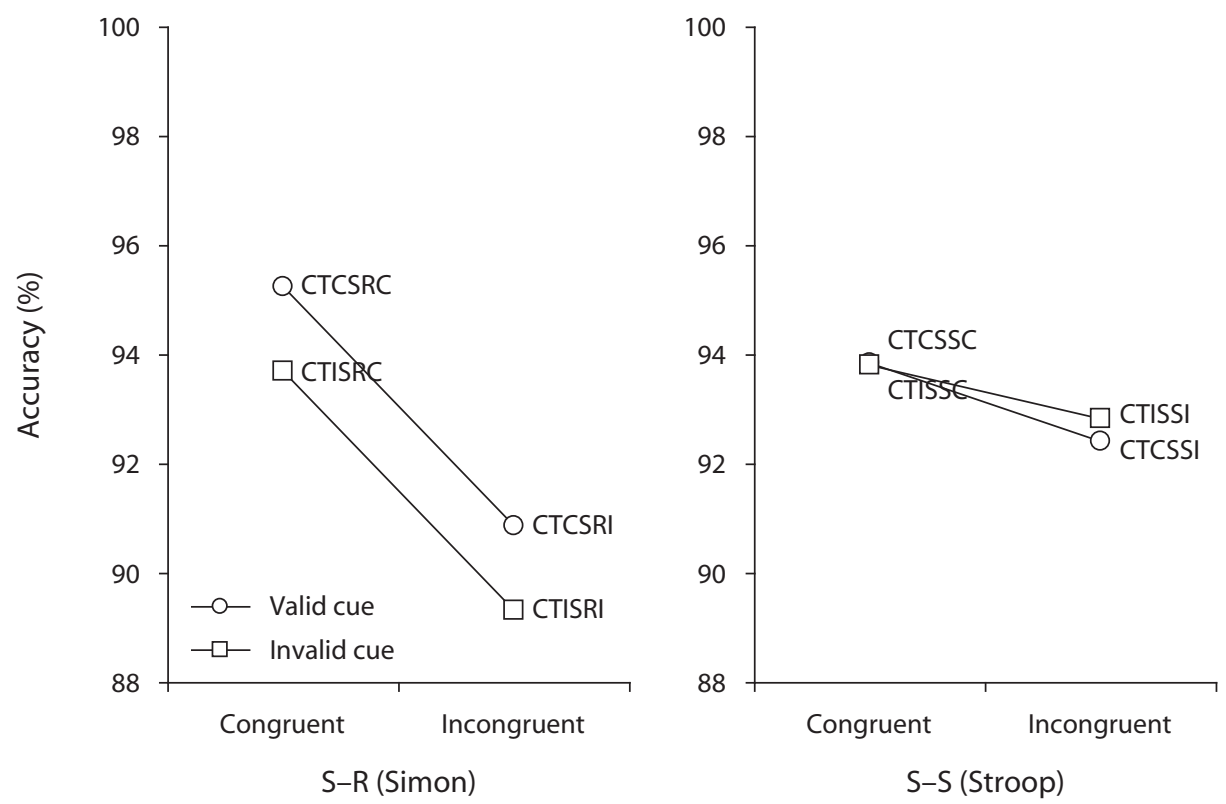

Figure 4. Reaction time (A) and accuracy (B) on the Simon-spatial-Stroop task.

$555, p<.05$; see Figure 5A]. However, the interaction was not a result of increased effect for dual sources of interference (i.e., CTISSI) but was a result of attenuated spatial Stroop interference following a valid cue (i.e., CTCSSI). For C-T $\times$ S-S analysis on accuracy, main effects for both $\mathrm{C}-\mathrm{T}$ and S-S compatibility effects were not significant $\left[\mathrm{C}-\mathrm{T}, F(1,29)=0.05, M S_{\mathrm{e}}=15, p>.05 ; \mathrm{S}-\mathrm{S}, F(1,29)=\right.$ $\left.3.05, M S_{\mathrm{e}}=14, p>.05\right]$. The interaction was not significant $\left[F(1,29)=0.17, M S_{\mathrm{e}}=10, p>.05\right.$; see Figure 5B].

Using the neutral condition as the baseline $(M=510$, $S D=82$ ), only double facilitation of the CTCSRC condi- tion showed an improvement of $29 \operatorname{msec}[t(29)=3.55$, $p<.005]$, whereas double interference of the CTISRI and CTISSI conditions significantly increased RT by $30 \mathrm{msec}$ $[t(29)=3.76, p<.005]$ and $44 \mathrm{msec}[t(29)=5.88, p<$ $.001]$, respectively. When there was a mixture of congruent and incongruent $\mathrm{C}-\mathrm{T}, \mathrm{S}-\mathrm{R}$, and $\mathrm{S}-\mathrm{S}$ overlaps (e.g., congruent $\mathrm{C}-\mathrm{T}$ with incongruent $\mathrm{S}-\mathrm{S}$; CTCSSI), the facilitation and interference effects seem to have canceled each other out and resulted in no significant improvement or impairment of performance, relative to the NEU baseline. 


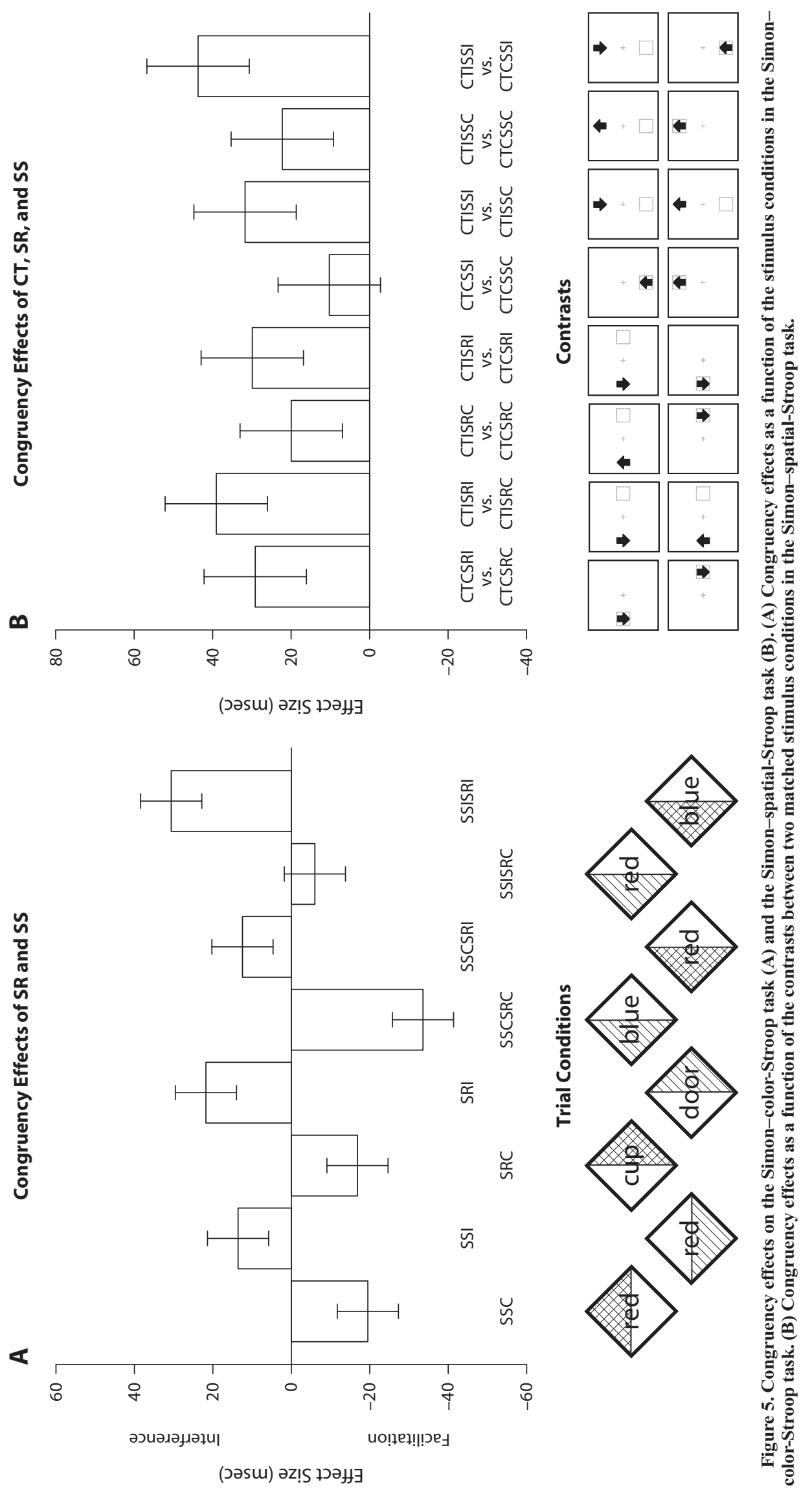


To break down the main effects of each dimensional overlap, Figure 4B presents pairwise comparisons of congruency when congruency of the other dimensions was kept constant. All but CTCSSI versus CTCSSC and CTISRC versus CTCSRC were significant by Bonferroni correction at $p<.05$.

None of the effects - S-S, S-R, and C-T - was significantly correlated with the others. There was no significant correlation among the effects across two tasks.

\section{DISCUSSION}

The present study provided behavioral evidence that DO captures various attentional effects. According to this theory, DO across $\mathrm{S}_{\mathrm{R}}, \mathrm{S}_{\mathrm{I}}$, and $\mathrm{R}$ determines $\mathrm{S}-\mathrm{S}$ and $\mathrm{S}-\mathrm{R}$ incompatibilities, which call upon attentional control. We also extended DO by increasing the number of dimensions beyond the original three $\left(\mathrm{S}_{\mathrm{R}}, \mathrm{S}_{\mathrm{I}}\right.$, and $\left.\mathrm{R}\right)$, since the $\mathrm{S}_{\mathrm{I}}$ dimensions can be more than one. As is illustrated in Figure 1, the Simon-color-Stroop task required individuals to respond to the color using a left or right buttonpress. Therefore, both the word enclosed in the diamond shape and the direction of the pointing angle of the colored triangle were $S_{I}$ dimensions, which would cause interference when they were incompatible with the $\mathrm{S}_{\mathrm{R}}$ dimension. The incongruent color word provided a source of $S_{R}-S_{I}$ incompatibility, whereas the colored triangle pointing to an opposite direction from the correct response constituted $\mathrm{S}_{\mathrm{I}}-\mathrm{R}$ incompatibility. In another scenario, when combining the cue validity manipulation and the spatial Stroop interference (Figure 2), DO took place between the direction of the arrow $\left(\mathrm{S}_{\mathrm{R}}\right)$ and cue location $\left(\mathrm{S}_{\mathrm{I}}\right)$ and between the direction of the arrow $\left(\mathrm{S}_{\mathrm{R}}\right)$ and target location $\left(\mathrm{S}_{\mathrm{I}}\right)$, as well as between the cue and target locations (both are $\mathrm{S}_{\mathrm{I}}$ ).

DO not only provides a universal framework for capturing various attentional effects acting alone, but also accounts for and makes predictions about the interactions of these attentional effects - for example, whether conflict effects resulting from various sources of S-S and S-R incompatibility are additive, subadditive, or superadditive or whether and in what way interferences from different sources (e.g., orienting and executive control) interact (e.g., amplify, overshadow, or cancel out one another). On the one hand, most of the results from both tasks implied independent processing of incompatibility rising from either S-S or S-R dimensional overlap. Interference effects resulting from $\mathrm{S}-\mathrm{S}$ and $\mathrm{S}-\mathrm{R}$ incompatibility within each task, as well as across both tasks, showed no correlation across participants. In addition, interference effects due to S-S and S-R incompatibility contributed additively when DO existed in both $\mathrm{S}-\mathrm{S}$ and $\mathrm{S}-\mathrm{R}$ ensembles, given the lack of interaction between $\mathrm{S}-\mathrm{S}$ and $\mathrm{S}-\mathrm{R}$ incompatibility. On the other hand, when opposite S-S and S-R effects were combined in the conditions-for example, incongruent S-S effect combined with congruent S-R effect (e.g., SSISRC in the Simon-color-Stroop task) - these effects seemed to interact with each other by canceling out overall facilitation or interference. Such interaction was also observed in the Simon-spatial-Stroop task when the interference effect by an incongruent spatial Stroop stimu- lus was canceled by a valid cue (i.e., RTs for CTCSSI and CTCSSC were not significantly different).

Furthermore, DO offers some insights into the common and distinct neural mechanisms of cognitive control involved in diverse attentional paradigms. Existing theories regarding the neural mechanisms of attention and its role in cognitive control have put much emphasis on the distinction among different components of the attentional network. For example, following the earlier work of Posner (Posner \& Petersen, 1990), the attention network test has identified three different subsystems of the attentional network-namely, alerting, orienting, and executive control (Fan, McCandliss, Fossella, Flombaum, \& Posner, 2005; Fan, McCandliss, Sommer, Raz, \& Posner, 2002). Another framework has been proposed to distinguish the roles of the top-down goal-directed attention pathway and the bottom-up stimulusinduced attention pathway (Corbetta, Kincade, Ollinger, McAvoy, \& Shulman, 2000; Corbetta \& Shulman, 2002). Yet another theory has focused on conflict monitoring and resolution, a more specific component of attentional functions (Botvinick, Braver, Barch, Carter, \& Cohen, 2001).

However, these specialized theories face several challenges, because they mostly focus on the distinct roles of individual components of the attentional system and, thus, fall short in accounting for the common driving force underlying various attentional effects. Several issues remain unsolved and call for further investigation. First, many neuroimaging studies have revealed a great deal of overlap, in terms of brain activation patterns, between the attentional process and other cognitive processes (e.g., working memory), of which the unique contribution of attention is hard to separate from that of other processes (Awh \& Jonides, 2001; Courtney, 2004). Second, although existing theories have attempted to distinguish different aspects of attentional functions and to identify distinct neural substrates that implement these processes, empirical studies have revealed that different attentional components recruit both common and distinct brain networks. For example, the frontoparietal network has been similarly activated by both orienting and executive control (Fan, Flombaum, McCandliss, Thomas, \& Posner, 2003; Hahn, Ross, \& Stein, 2006; Kincade, Abrams, Astafiev, Shulman, \& Corbetta, 2005; Liu et al., 2004; Liu, Banich, Jacobson, \& Tanabe, 2006; Vossel, Thiel, \& Fink, 2006). Moreover, according to meta-analyses of a large number of neuroimaging studies of attention and interference resolution (Nee, Wager, \& Jonides, 2007; Wager, Jonides, \& Reading, 2004; Wager et al., 2005), many of the discrepancies in brain activation patterns result from differences in recruited participant samples, task paradigms, experimental designs, and analytic approaches, rather than reflecting genuine distinctions in attentional processes and underlying neural substrates. DO can help bridge the gap between empirical findings and existing theories. For example, by applying the present tasks developed under the guidance of this general framework in neuroimaging studies, which has been planned, we will be able to examine common and distinct neural substrates that underlie diverse attentional effects resulting from various $\mathrm{S}-\mathrm{S}$ and $\mathrm{S}-\mathrm{R}$ dimensional overlaps and elucidate whether a single or multiple conflict detection 
and/or executive control networks exist in the brain (Egner, 2008). Future studies can also examine whether DO can predict and/or account for cross-modal attentional effects when DOs come from different modalities (e.g., combining auditory cues and visual/spatial targets), and whether incompatibility effects resulting from cross-modal DOs are reduced, as compared with when DOs come from a single modality (e.g., visual stimuli).

\section{AUTHOR NOTE}

This study was supported by National Center for Research Resources (NCRR) Grant MO1 RR00071 and a NARSAD Young Investigator Award (to X.L.). The authors thank Michael I. Posner for insightful comments. Correspondence concerning this article should be addressed to X. Liu, Institute of Psychology, Chinese Academy of Sciences, 4A Datun Road, Beijing 100101, China (e-mail: liux@psych.ac.cn).

\section{REFERENCES}

AwH, E., \& Jonides, J. (2001). Overlapping mechanisms of attention and spatial working memory. Trends in Cognitive Sciences, 5, 119126. doi:10.1016/S1364-6613(00)01593-X

Banich, M. T., Milham, M. P., Atchley, R., Cohen, N. J., WebB, A., WszaleK, T., ET AL. (2000). fMRI studies of Stroop tasks reveal unique roles of anterior and posterior brain systems in attentional selection. Journal of Cognitive Neuroscience, 12, 988-1000. doi:10.1162/08989290051137521

Botvinick, M. M., Braver, T. S., Barch, D. M., Carter, C. S., \& Cohen, J. D. (2001). Conflict monitoring and cognitive control. Psychological Review, 108, 624-652. doi:10.1037/0033-295X.108.3.624

Bush, G., Whalen, P. J., Rosen, B. R., Jenike, M. A., McInerney, S. C., \& Rauch, S. L. (1998). The counting Stroop: An interference task specialized for functional neuroimaging - Validation study with functional MRI. Human Brain Mapping, 6, 270-282. doi:10.1002/ (SICI) 1097-0193(1998)6:4<270::AID-HBM6>3.0.CO;2-0

Corbetta, M., Kincade, J. M., Ollinger, J. M., McAvoy, M. P., \& Shulman, G. L. (2000). Voluntary orienting is dissociated from target detection in human posterior parietal cortex. Nature Neuroscience, $\mathbf{3}$, 292-297. doi:10.1038/73009

Corbetta, M., \& Shulman, G. L. (2002). Control of goal-directed and stimulus-driven attention in the brain. Nature Reviews Neuroscience, 3, 201-215. doi:10.1038/nrn755

Courtney, S. M. (2004). Attention and cognitive control as emergent properties of information representation in working memory. Cognitive, Affective, \& Behavioral Neuroscience, 4, 501-516. doi:10.3758/ CABN.4.4.501

Desimone, R., \& Duncan, J. (1995). Neural mechanisms of selective visual attention. Annual Review of Neuroscience, 18, 193-222. doi:10.1146/annurev.ne.18.030195.001205

EGNER, T. (2008). Multiple conflict-driven control mechanisms in the human brain. Trends in Cognitive Sciences, 12, 374-380. doi:10.1016/ j.tics.2008.07.001

Fan, J., Flombaum, J. I., McCandliss, B. D., Thomas, K. M., \& PosNER, M. I. (2003). Cognitive and brain consequences of conflict. NeuroImage, 18, 42-57. doi:10.1006/nimg.2002.1319

Fan, J., McCandliss, B. D., Fossella, J., Flombaum, J. I., \& Posner, M. I. (2005). The activation of attentional networks. NeuroImage, 26, 471-479. doi:10.1016/j.neuroimage.2005.02.004

Fan, J., McCandliss, B. D., Sommer, T., Raz, A., \& Posner, M. I. (2002). Testing the efficiency and independence of attentional networks. Journal of Cognitive Neuroscience, 14, 340-347. doi:10.1162/089892902317361886

Hahn, B., Ross, T. J., \& Stein, E. A. (2006). Neuroanatomical dissociation between bottom-up and top-down processes of visuospatial selective attention. NeuroImage, 32, 842-853. doi:10.1016/ j.neuroimage.2006.04.177

Kincade, J. M., Abrams, R. A., Astafiev, S. V., Shulman, G. L., \& Corbetta, M. (2005). An event-related functional magnetic resonance imaging study of voluntary and stimulus-driven orienting of attention. Journal of Neuroscience, 25, 4593-4604. doi:10.1523/ jneurosci.0236-05.2005
Knudsen, E. I. (2007). Fundamental components of attention. An nual Review of Neuroscience, 30, 57-78. doi:10.1146/annurev.neuro .30.051606.094256

Kornblum, S., HasbroucQ, T., \& Osman, A. (1990). Dimensional overlap: Cognitive basis for stimulus-response compatibility-A model and taxonomy. Psychological Review, 97, 253-270. doi:10.1037/0033 $-295 X .97 .2 .253$

Kornblum, S., \& LeE, J. W. (1995). Stimulus-response compatibility with relevant and irrelevant stimulus dimensions that do and do not overlap with the response. Journal of Experimental Psychology: Human Perception \& Performance, 21, 855-875. doi:10.1037/0096 $-1523.21 .4 .855$

Kornblum, S., Stevens, G. T., Whipple, A., \& Requin, J. (1999). The effects of irrelevant stimuli: 1 . The time course of stimulus-stimulus and stimulus-response consistency effects with Stroop-like stimuli, Simon-like tasks, and their factorial combinations. Journal of Experimental Psychology: Human Perception \& Performance, 25, 688-714. doi:10.1037/0096-1523.25.3.688

Liu, X., Banich, M. T., Jacobson, B. L., \& Tanabe, J. L. (2004). Common and distinct neural substrates of attentional control in an integrated Simon and spatial Stroop task as assessed by event-related fMRI. NeuroImage, 22, 1097-1106. doi:10.1016/j.neuroimage.2004 .02 .033

LiU, X., Banich, M. T., Jacobson, B. L., \& Tanabe, J. L. (2006). Functional dissociation of attentional selection within PFC: Response and non-response related aspects of attentional selection as ascertained by fMRI. Cerebral Cortex, 16, 827-834. doi:10.1093/cercor/bhj026

LiU, X., Wang, H., Corbly, C. R., Zhang, J., \& Joseph, J. E. (2006). The involvement of the inferior parietal cortex in the numerical Stroop effect and the distance effect in a two-digit number comparison task. Journal of Cognitive Neuroscience, 18, 1518-1530. doi:10.1162/ jocn.2006.18.9.1518

MacLeod, C. M. (1991). Half a century of research on the Stroop effect: An integrative review. Psychological Bulletin, 109, 163-203. doi:10.1037/0033-2909.109.2.163

MacLeod, C. M. (1992). The Stroop task: The "gold standard" of attentional measures. Journal of Experimental Psychology: General, 121, 12-14. doi:10.1037/0096-3445.121.1.12

NeE, D. E., Wager, T. D., \& Jonides, J. (2007). Interference resolution: Insights from a meta-analysis of neuroimaging tasks. Cognitive, Affective, \& Behavioral Neuroscience, 7, 1-17. doi:10.3758/CABN.7.1.1

Posner, M. I., Cohen, Y., \& RafaL, R. D. (1982). Neural systems control of spatial orienting. Philosophical Transactions of the Royal Society $B, \mathbf{2 9 8}, 187-198$. doi:10.1098/rstb.1982.0081

Posner, M. I., \& Petersen, S. E. (1990). The attention system of the human brain. Annual Review of Neuroscience, 13, 25-42. doi:10.1146/ annurev.ne.13.030190.000325

Posner, M. I., SNyder, C. R., \& Davidson, B. J. (1980). Attention and the detection of signals. Journal of Experimental Psychology, 109, 160-174.

Proctor, R. W., \& Reeve, T. G. (Eds.) (1990). Stimulus-response compatibility: An integrated perspective. Amsterdam: North-Holland.

SimON, J. R., \& SMALL, A. M., JR. (1969). Processing auditory information: Interference from an irrelevant cue. Journal of Applied Psychology, 53, 433-435.

STROOP, J. R. (1935). Studies of interference in serial verbal reactions. Journal of Experimental Psychology, 18, 643-662.

Vossel, S., Thiel, C. M., \& Fink, G. R. (2006). Cue validity modulates the neural correlates of covert endogenous orienting of attention in parietal and frontal cortex. Neurolmage, 32, 1257-1264. doi:10.1016/ j.neuroimage.2006.05.019

WAGER, T. D., JoNIDES, J., \& READING, S. (2004). Neuroimaging studies of shifting attention: A meta-analysis. NeuroImage, 22, 1679-1693. doi:10.1016/j.neuroimage.2004.03.052

Wager, T. D., Sylvester, C. Y., Lacey, S. C., Nee, D. E., FrankLIN, M., \& Jonides, J. (2005). Common and unique components of response inhibition revealed by fMRI. NeuroImage, 27, 323-340. doi:10.1016/j.neuroimage.2005.01.054

Zhang, H., \& Kornblum, S. (1998). The effects of stimulus-response mapping and irrelevant stimulus-response and stimulus-stimulus overlap in four-choice Stroop tasks with single-carrier stimuli. Journal of Experimental Psychology: Human Perception \& Performance, 24, 3-19. doi:10.1037/0096-1523.24.1.3 


\section{APPENDIX}

Table A1

Conditional Labels, Means, and Standard

Deviations of the Response Times (RTs, in

Milliseconds) and Accuracy (As a Percentage) for the Simon-Color-Stroop Task

\begin{tabular}{lccccc}
\hline & \multicolumn{2}{c}{ RT } & & \multicolumn{2}{c}{ Accuracy } \\
\cline { 2 - 3 } \cline { 6 - 6 } Condition & $M$ & $S D$ & & $M$ & $S D$ \\
\hline NEU & 473 & 58 & & 97.87 & 2.64 \\
SSC & 454 & 54 & & 97.60 & 3.40 \\
SSI & 487 & 58 & & 96.77 & 3.18 \\
SRC & 456 & 59 & & 98.73 & 2.00 \\
SRI & 495 & 67 & & 95.47 & 5.56 \\
SSCSRC & 440 & 59 & & 98.40 & 2.49 \\
SSCSRI & 486 & 64 & & 96.60 & 4.32 \\
SSISRC & 467 & 54 & & 97.67 & 2.92 \\
SSISRI & 504 & 60 & & 94.77 & 5.35 \\
\hline
\end{tabular}

Table A2

Behavioral Effects of the Response Times (in Milliseconds) for the Simon-Color-Stroop Iask

\begin{tabular}{|c|c|c|c|c|c|c|}
\hline & \multicolumn{3}{|c|}{ Facilitation } & \multicolumn{3}{|c|}{ Interference } \\
\hline & \multicolumn{2}{|c|}{ Single } & \multirow{2}{*}{$\frac{\text { Double }}{\text { SSCSRC }}$} & \multicolumn{2}{|c|}{ Single } & \multirow{2}{*}{$\frac{\text { Double }}{\text { SSISRI }}$} \\
\hline & $\mathrm{SSC}$ & $\mathrm{SRC}$ & & SSI & SRI & \\
\hline$M$ & -20 & -17 & -34 & 14 & 22 & 31 \\
\hline$S D$ & 22 & 22 & 22 & 27 & 29 & 24 \\
\hline$t$ & -4.86 & -4.18 & -8.31 & 2.78 & 4.11 & 6.99 \\
\hline$p$ & .00 & .00 & .00 & .01 & .00 & .00 \\
\hline
\end{tabular}

Table A3

Conditional Labels, Means, and Standard Deviations of the Response Times (RTs, in Milliseconds) and Accuracy (As a Percentage) for the Simon-Spatial-Stroop Task

\begin{tabular}{lccccr}
\hline & \multicolumn{2}{c}{ RT } & & \multicolumn{2}{c}{ Accuracy } \\
\cline { 2 - 3 } \cline { 6 - 6 } Condition & $M$ & $S D$ & & $M$ & $S D$ \\
\hline NEU & 510 & 82 & & 94.50 & 11.03 \\
CTCSRC & 481 & 59 & & 95.23 & 9.76 \\
CTCSRI & 510 & 68 & & 90.87 & 11.33 \\
CTISRC & 501 & 76 & & 93.70 & 10.29 \\
CTISRI & 540 & 77 & & 89.33 & 12.20 \\
CTCSSC & 500 & 70 & & 93.87 & 10.64 \\
CTCSSI & 510 & 70 & & 92.43 & 10.58 \\
CTISSC & 522 & 67 & & 93.80 & 10.21 \\
CTISSI & 554 & 64 & & 92.83 & 9.55 \\
\hline
\end{tabular}

Table A4

Behavioral Effects of the Response Times (in Milliseconds) for the Simon-Spatial-Stroop Task

\begin{tabular}{|c|c|c|c|c|c|c|c|c|}
\hline & \multicolumn{2}{|c|}{ CTC } & \multicolumn{2}{|c|}{ CTI } & \multirow{2}{*}{\multicolumn{4}{|c|}{ CTI vs. CTC }} \\
\hline & SRI vs. & SSI vs. & SRI vs. & SSI vS. & & & & \\
\hline & $\mathrm{SRC}$ & SSC & SRC & SSC & $\mathrm{SRC}$ & SRI & $\mathrm{SSC}$ & SSI \\
\hline$M$ & 29 & 10 & 39 & 32 & 20 & 30 & 22 & 44 \\
\hline$S D$ & 36 & 28 & 43 & 42 & 43 & 32 & 28 & 44 \\
\hline$t$ & 4.39 & 1.98 & 5.01 & 4.14 & 2.56 & 5.06 & 4.31 & 5.43 \\
\hline$p$ & .00 & .06 & .00 & .00 & .02 & .00 & .00 & .00 \\
\hline
\end{tabular}

(Manuscript received February 5, 2010; revision accepted for publication April 16, 2010.) 